\title{
VALORACIÓN CIENTÍFICA DEL LITORAL ALICANTINO ${ }^{1}$ \\ Vicenç M. Rosselló i Verger
}

\begin{abstract}
RESUMEN
El artículo se refiere al ámbito del antiguo «terme general d'Alacant», desde el Campello hasta el Carabassí, donde los accidentes costeros responden a la tectónica pliopleistocénica. La escasez del aporte sedimentario da mayor beligerancia al factor oleaje que depende de un predominio de los vientos SE y E. En los sectores bajos las playas han visto eliminar las dunas y las albuferas han sido transformadas. De los sectores medios o altos destaca el karst marino y los yacimientos cuaternarios, estudiados y revisitados desde 1906 y, sobre todo, desde 1955 y 1957 (Congreso INQUA); su principal rasgo es el nivel fundamental eutirreniense de fauna cálida (Strombus). La artificialización del litoral ha supuesto la construcción del puerto de Alicante, uno de los más antiguos del País Valenciano, la implantación de caminos litorales y la instalación de diques y espigones de defensa. Se recuerda la oportunidad de salvaguardar las atalayas del siglo XVII.
\end{abstract}

\begin{abstract}
The paper refers to the scope of the old «terme general d'Alacant», from el Campello to el Carabassí, where the coastal accidents respond to the pliopleistocene tectonics. The shortage of sedimentary contribution gives greater role to the waves factor which depends on the predominance of the SE and E winds. On low sectors the beaches have seen disappear the dunes and the lagoons have been changed. From medium of high shorelines are to point out the marine karst and the Quaternary deposits, studied and checkec since 1906, mainly since 1955 and 1957 (INQUA Congress); its main feature is the Euthyrhenian fundamental level of warm fauna (Strombus). The littoral has become artificial by means of building a port in Alacant, one of the oldest in Valencian Land, the establishment of littoral promenades and the installation of groins and jetties. It is reminded the opportunity to save the watch-towers from the XVII ${ }^{\text {th }}$ century.
\end{abstract}

Fijar de entrada el alcance de esta ponencia exige resolver una alternativa. ¿Se trata de la enumeración de los valores científicos que hay en el litoral aludido o de la valoración

${ }^{1}$ Texto literal de la conferencia pronunciada el día 23 de noviembre de 1989, dentro del ciclo «Alicante, una ciudad hacia el futuro», desarrollado con motivo del Quinto Centenario de la Ciudad de Alicante. 
que hace un científico de ese litoral? Más objetivo, al parecer, el primer enfoque, más subjetivo el segundo. Para decir que me gusta -o me disgusta- el litoral alicantino, tal vez no hubiera venido; por ello me esforzaré en destacar el valor científico -los valores científicos, naturales sobre todo- y los peligros que acechan un sector de costa muy querido por todos los componentes de esta mesa.

Una segunda precisión se refiere al alcance del gentilicio: ¿municipio, comarca, provincia? Uno que es poco entusiasta del provincianismo y las provincias, ha optado por el antiguo terme general o particular contribución que en el litoral coincide grosso modo con la comarca de l'Alacantí; o sea, Santa Pola y la Vila Joiosa quedarán fuera de nuestra consideración.

\section{Los factores naturales}

La osamenta del relieve hay que buscarla en la estructura, en nuestro caso, en los plegamientos subbéticos que pueden responsabilizarse directamente de los acantilados del $\mathrm{N}$ del Campello. La tectónica también responde de la línea quebrada en dos rectángulos de los Caps de l'Horta y de l'Aljup, pero bajando a más detalle en el primero encontramos un islote trapezoidal mioceno que emerge del cuaternario, así como la tabla inclinada miopliocena o tal vez domo de la Serra de Santa Pola. Un abrupto, aunque pequeño, anticlinal cizallado, también mioceno, forma la Serra Grossa o de Sant Julià, de orientación E-W, al sudoeste de la cual fue vaciado para construir el nuevo puerto el Tossal del Molinet. Al S de la ciudad la tectónica postpliocena se manifiesta en ejes transversales a la costa que han repercutido en la desnivelación del pleistoceno. Aparte queda, no hay que olvidarlo, la Illa Plana o Nova Tabarca, extremo del plegamiento penibético y no precisamente volcánica, como se ha repetido.

$\mathrm{Al}$ no ser tan conspicua y constante en este tramo litoral la deriva que se registra en buena parte de la costa valenciana, hay que atender como agente marino primordial el oleaje y su causa, el viento. El observatorio de Alacant arroja un predominio del E y SE entre los vientos marinos, pero una bonanza frecuente. En el de l'Altet, en cambio, el levante destaca mucho más claramente, en todo lo cual influye la abundancia de las brisas. Los fuertes temporales sólo llegan como mar de fondo, aunque se recuerden episodios como el del Campello en diciembre de 1980, desgraciadamente memorables. Los fetchs máximos -es decir los recorridos de los vientos que engendran oleajes- corresponden al SE $(974 \mathrm{~km})$ y E $(1.384 \mathrm{~km})$, precisamente los más frecuentes; en tales casos la altura máxima de las olas puede alcanzar los 6 ó $7 \mathrm{~m}$ «Mano de obra» no falta para el modelado litoral.

Lo que sí escasea es el material, el material móvil, al menos en algunos sectores. No hay ningún río importante que pueda responsabilizarse del suministro. El más caudaloso es el Riu Verd, de Castalla, de Montnegre que al desembocar -valga la ironía- se llama Riu Sec. Es una auténtica rambla con un módulo de $0,27 \mathrm{~m}^{3} / \mathrm{seg}$ que no excluye fuertes y distantes avenidas. Menor papel tiene todavía a nuestros efectos la inconexa Rambla de les Ovelles. En virtud de lo dicho puede pensarse que la movilización de sedimentos resultará más importante que el aporte de material nuevo.

\section{Costas de erosión}

Un litoral relativamente variado permite distinguir dos grandes tipos, con valiosos 
detalles a veces. El primero lo forman las costas de erosión, acantilados o costas altas, no por desconocidas, menos interesantes.

El acantilado alto del litoral longitudinal se inicia con el Tossal del Mar y se prolonga por la Venta de Lanuza, con más de $100 \mathrm{~m}$ de pared casi vertical y acarcavada. Después de Cala Lanuza y Cala Baeza se eleva de nuevo y alcanza casi los $200 \mathrm{~m}$ en les Llomes de Reixes (o Rejàs), cortadas por barrancos suspendidos al parecer a causa de un enérgico retroceso erosivo. La debilidad de los materiales eocénicos permite la formación de una rasa marina amplia. Al S de la Coveta Fumada -ya de acantilado medio- la plataforma de abrasión se suelda a una acera de eolianitas pleistocenas con avanzado modelado cárstico como el de la Punta del Llop Marí o la Illeta dels Banyets.

Cabe considerar también la costa baja rocosa del Cap de l'Horta, importante en detalles cuaternarios, y el acantilado muy antropizado, por no decir artificial, de las molasas miocenas de la Serra Grossa o de Sant Julià. Para acabar se habría de saltar al acantilado fósil del domo mioplioceno de Santa Pola, perteneciente ya al Baix Vinalopó.

\section{El karst marino}

La disolución de la caliza por la acción del agua marina ayudada por mecanismos bioquímicos es un rasgo peculiar que marca ciertos segmentos de nuestro litoral. Lo encontramos al pie de la Serra dels Colmenars, entre la Cala dels Borratxos y Cala Barda, aunque el desplome frecuente del modesto frente litoral no deja demasiado margen para su desarrollo. El entorno de l'Albufereta conservaba un pequeño retazo con pozas de 15 a $35 \mathrm{~cm}$ de diámetro, poco vistas, y algunos bufones o bufadors. Otro pequeño sector radica -o radicaba- en la Cala dels Jueus.

El Cap de l'Horta en conjunto muestra una banqueta mesolitoral de considerable extensión y una zona supralitoral donde afloran pequeños diques de hasta $20 \mathrm{~m}$ de longitud que vienen a ser crestas monoclinales en miniatura. Cuencos y pilancones abundan en todo el perímetro. La Illeta dels Banyets, porción desgajada de 220 x 70 m, muestra un abundante lapiaz denticular, cuencos, cubetas y balmas que pueden correlacionarse con canteras de muelas y sillares y un establecimiento pesquero romano.

\section{Costas de acumulación}

Los litorales bajos, que suelen coincidir con postpaís deprimido o llano, son casi siempre playas y a raíz de la cultura del ocio son mucho más visitados, accesibles y codiciados.

La plataforma continental frente a las costas alicantinas alcanza unos $45 \mathrm{~km}$ de anchura; en otras palabras, el gradiente del fondo antelitoral es en un gran espacio de sólo $0,3 \%$, lo cual favorece la acumulación de sedimentos. Éstos han sido rigurosamente estudiados por E. Sanjaume (1985) que ha deducido, a diferencia de lo que se produce en el óvalo norvalenciano, una cierta incomunicación litoral.

Al $\mathrm{N}$ del término del Campello tropezamos con el primer canturral en la Platja del Carritxar $\mathrm{y}$, después de un largo tramo de costa estructural acantilada, viene otra playa de cantos con alguna proporción de arena en el Carrer del Mar del Campello, tal vez debida a la antigua aportación fluvial del Riu Sec. La misma protuberancia de este río aparece orlada de un canturral de fácil interpretación.

En cambio, la Platja de l'Horta o de Sant Joan -de «Muchavista»- es arenosa y 
procede más bien de autoalimentación a partir del ataque del Cap de l'Horta. Sabemos que en el siglo XVIII tenía una doble o triple fila de dunas... L'Albufereta encabezaba una playa de arena, hoy difícil de localizar, mientras la del Postiguet, antes de la implantación portuaria, se extendía más de un kilómetro, aunque su alimentación continúa siendo difícil, pese a estar a resguardo de los diques del puerto. En la Cala dels Borratxos y «Urbanova» predomina la arena intermedia, poco evolucionada, mientras que en el frente del Carabassí hay una gran afinidad entre la arena actual y la «fósil», es decir la litificada de las eolianitas o de las playas antiguas.

Con una óptica mineralógica, desde el Campello al Postiguet hay un predominio de la combinación dolomita-turmalina, relacionable con el abastecimiento del Riu Sec o de Montnegre. Más al sur, la turmalina sola.

El único accidente acumulativo destacable es el falso delta, mejor, cono del Riu Sec. Con una superficie de unas 50 ha (mayor en el siglo XVIII, si hemos de fiarnos de la cartografía), conforma un abanico de acarreos recientes que no encontramos, por contra, en el Barranc de les Ovelles, de desembocadura más problemática.

Uno de los rasgos más característicos del litoral valenciano, las albuferas, tampoco pueden estar ausentes. En un caso ya apenas es más que un topónimo: l'Albufereta. Pero no sólo fue, según afirman los arqueólogos, puerto romano, sino que en siglos recientes (Alberola, 1989) planteó serios problemas de salubridad como tantos otros aguazales mediterráneos. L'Albufera d'Elx queda fuera de nuestro conspectus, pero aquí podemos aludir al Salobrar d'Aigua Amarga, limítrofe, a 4,5 km al S de la ciudad y al pie de la Serra dels Colmenars. Su existencia guarda relación con la depresión de els Bassars-el Clot de Galvany y con la terraza marina flandriense estudiada por V. Gozálvez (1985).

\section{El pleistoceno marino}

Si hay un detalle alicantino de resonancia científica internacional es el de los niveles marinos cuaternarios que dejaron numerosos testimonios en esta costa. Son multitud los investigadores que desde principios de siglo han desfilado por los yacimientos, perseguidos con saña por la especulación inmobiliaria.

Inicia la serie el incansable investigador murciano Daniel Jiménez de Cisneros, que ocupó la cátedra de Ciencias Naturales del Instituto de Enseñanza Media de Alacant desde 1903. Hay numerosas referencias a la cuestión en la larguísima colección de sus artículos. En 1906 dio a conocer las «playas fósiles» de l'Albufereta y otras de los alrededores de Alacant. En la Geografía general del Reino de Valencia (1912 ca.) dirigida por F. Carreras habla (I, p. 411) de playas elevadas -que atribuye a epirogenia o basculación de la Península, pero no a eustatismoal pie de la Serra Grossa de Sant Julià. El depósito de la playa de l'Albufereta «levantado muchos metros sobre el nivel actual» se convertirá en una de las pistas de los futuros estudiosos. La aportación más categórica es el encuentro del Strombus bubonius en el subsuelo de la ciudad (1925) que, de todos modos, parece referirse a un ejemplar recogido en 1877 y que figuraba en la colección del Instituto sin apenas especificaciones. (Los strombi, ya los había citado un esforzado naturalista aragonés, el escolapio L. Calvo en 1908).

Pedro Novo (1915), en una aportación «oficial» de los ingenieros de minas al mapa geológico, se refiere a la tosca que emparenta con los médanos actuales y la localiza (entre otras) en l'Albufereta y el Cap de l'Horta. Más trascendental es la referencia a la playa levantada de +25 m en la ladera de la Serra de Sant Julià que mira hacia l'Albufereta: un yacimiento de casi $1 \mathrm{~km}$ x $100 \mathrm{~m}$, de caliza y molasas con marmitas. 
El primer extranjero -de una retahíla que acudirían sucesivamente- que interviene en el asunto del cuaternario marino valenciano parece que fue Marcel Gignoux en una nota de 1911 sobre los S. bubonii del Mediterráneo occidental, presentada a la Academia de Ciencias de París. Paul Fallot, en colaboración con el anterior (1922), reincidió en el tema del cuaternario marino. Guiados por Jiménez de Cisneros visitaron el yacimiento de l'Albufereta del que clasificaron unas cincuenta especies: Conustestudinarius, Tritonidea viverrata, Strombus bubonius y Natica lactea son las principales encontradas a $+15 \mathrm{~m}$, que les hicieron inferir un nivel marino de unos $+30 \mathrm{~m}$.

Con ocasión del XVI Congreso Geológico Internacional se celebró una excursión a nuestras costas cuya guía redactaron Gignoux y Fallot (1927). Un discípulo de Jiménez de Cisneros, Lincoln Albricias, presentó una destacada contribución al estudio de las playas elevadas de Alacant (1927). El avance de la investigación -que no se acabó- contiene extremos muy interesantes sobre los yacimientos del Cap de l'Horta, l'Albufereta, junto a les Cases de l'Almadrava, Cales de les Figueres y dels Capellans, formados por arenas rojizas con moluscos pleistocenos. El autor no se pronuncia por la epirogenia o la retirada del mar $\mathrm{y}$, aunque menciona los pisos de Depéret, no se compromete.

E. Hernández Pacheco (que había estimulado el trabajo de Albricias) volvió a citar los yacimientos de Alacant en el Congreso de la Asociación Española para el Progreso de las Ciencias de Lisboa (1932), destacando la rasa de la Illa Plana o Nova Tabarca y sus yacimientos tirrenienses. Este piso de facies someras y fauna termófila será el más caracterizado.

En 1955 sale un trabajo de Gigout, Solé y Solé, avance de investigaciones previas al Congreso Internacional del Cuaternario (INQUA), donde, bajo el equívoco título «Cuaternario mediterráneo de Andalucía», se detalla como flandriense la lumaquela a $2 \mathrm{~km} \mathrm{~W}$ del Cap de l'Horta con una dudosa atribución de Strombus, probablemente rodado. El tirreniense reciente (Ouljien) está más repetido y contiene, a veces, Strombus: se trata de un depósito cementado de playa que soporta dunas consolidadas y suele situarse a más de $+5 \mathrm{~m}$, p.e. en la ladera de la Serra Grossa, extremo W de l'Albufereta, Cap de l'Horta y Carrer del Mar del Campello; no suele estar deformado. Las trazas del tirreniense «antiguo» son mediocres, p.e. en l'Albufereta a +25 $m$ y en el extremo S de la Platja de Sant Joan. No contiene Strombus.

El Congreso INQUA 1957 acogió la excursión L cuyo libro guía fue dirigido por LI. Solé. Porta publicó un artículo sobre bioestadística de la fauna malacológica del Cap de l'Horta en 1958. Leo Imperatori, ingeniero industrial aficionado a la geología, estudió en sendos artículos $(1957,1959)$ los yacimientos del flanco S del Cap de l'Horta, a punto de ser destruidos, y la parte oriental de l'Albufereta, en la bajada al mar desde el cuartel de carabineros ya desaparecido como la mayoría de testigos. También procede del Congreso la nota de Gigout, Solé y Solé (1957) sobre la Serra dels Colmenars que conserva restos de «probable calabriense» por encima (+90 m) del acantilado muerto «ouljiense» que mira al mar en el confín entre Alacant y Elx. Gigout (1962), que se obstina en hacer andaluces Alacant y Altea, admite dos niveles de Strombus, tirrenienses, pero se empecina en el erróneo hallazgo de la especie en el «versiliense» del Cap de l'Horta. En aquel momento se conocían 200 especies tirrenienses en la comarca alicantina.

Un artículo renovador de Steams y Thurber (1965) aplica la datación readioisotópica (Pa 231/Th 230) a tres muestras del Cap de l'Horta, entre otras, Glycymeris violacescens del tirreniense I al S de la Platja de Sant Joan (+18 m,> 200.000 BP), tirreniense II del Cap de l'Horta a +7 m (85.000 BP), y tirreniense III, más bajo, 33.000 BP. Pese al actual descrédito de la radiocronología, las referidas fechas grosso modo se mantienen.

Los autores franceses no suelen admitir más división que la de paleotirreniense y 
eutirreniense. Así Montenat (1973) atribuye al eutirreniense los yacimientos de los Arenals del Sol y al tirreniense indiferenciado los de $+10 \mathrm{~m}$ de la Illa Plana, mientras la caliza oolítica de las Salinas Catalanas (S de Alacant) la da como eutirreniense. Por otro lado, B. Dumas (1977) llama la atención sobre la deformación del paleotirreniense en el Cap de l'Horta y l'Albufereta al estudiar los yacimientos en estas localidades y en el Carrer del Mar del Campello. Da cuenta de nuevos hallazgos al S del Benacantil y separa claramente dos tirrenienses, uno de $221.000 \mathrm{BP}$ y otro del 85.000, descartando sistemáticamente el neotirreniense. Alude a varias playas tirrenienses al pie de la Serra dels Colmenars (1981) y al sur del Carabassí, donde distingue cuatro tipos de duna; recalca la neotectónica del Cap de l'Horta y l'Albufereta donde ha actuado el juego de la compresión y desenganche.

C. Gaibar trabajó primero en Nova Tabarca $(1965,1972)$ donde recogió 2.555 fósiles que serían clasificados por J. Cuerda y luego en el Molar y els Colmenars (1969). Fueron toneladas de material malacológico las que acumuló y, con una extraña combinación de desconfianza y prudencia científica, ocultaba las localidades de hallazgo a su imprescindible colaborador. Un implacable trabajo de adalid o desbrozador no dejó un decímetro por explorar: 15.300 moluscos fósiles recogió en el Cap de l'Horta. Dejando aparte la danza de números con que pretende explicar los hechos neotectónicos y eustáticos, confirmó sólidamente el tirreniense II (p.e. en Cala Ribelles, ahora destruido) del flanco N del Cap de l'Horta, mientras en el flanco S atribuía los niveles altos al «calabriense».

Valga la extensa recensión como indicio del papel que un litoral puede asumir en el desarrollo científico. No entro en su riqueza biológica; otros especialistas podrán hacerlo mejor.

\section{La antropización}

Un primer intento de dominio de la costa se materializa en el afán de su defensa y ataque. Contra la piratería se levantan castillos y torres. El de Santa Bàrbera desde buena parte de su estructura más visible al Marqués de Castelo Rodrigo a fines del siglo XVII. Las torres de costa que combinaban la vigilancia con las compañías de caballos no son muy anteriores; nos atañen ahora y aquí la Torre de l'Aigua, la Torre de la Illeta, la Torre del Cap de l'Horta, la Torre de l'Aigua Amarga, la Torre Carabassina y la del Cap de l'Aljup. ¿Se ha preocupado alguien de salvar lo salvable de tales monumentos? Si trataron de protegernos durante dos o tres siglos, justo será dos o tres siglos después que los protejamos, antes de que sea demasiado tarde.

Otra transformación antrópica de un cierto interés son las salinas. Las únicas que afectan el territorio acotado, abandonadas hace más de veinte años, son las del Saladar d'Aigua Amarga ¿podría salvarse algo de su interesantísimo entorno, acechado por Urbanova, Gran Alacant, Arenals del Sol? Dejo de lado, por conocido, el problema de los humedales del Clot de Galvany. Alguien más autorizado que yo podrá referirse a ellos en el coloquio.

En otro lugar (Rosselló, 1986) me he referido a la artificialización del litoral valenciano. Trataré de reasumir aquí lo aplicable al sector alicantino. A los usos «industriales», pesqueros, de puertos comerciales, hay que unir los «turístico-recreativos» que ahora suman a la ocupación urbana la frecuente implantación rururbana de una franja de 2 ó $3 \mathrm{~km}$-y hasta 5 ó 6- por edificios dedicados al ocio o a la segunda vivienda. Las mal llamadas «urbanizaciones» se han extendido con carácter explosivo, sobre todo durante los años 1970 en nuestro litoral (Gozálvez, 1984). 


\section{Los puertos}

La historia del de Alacant es de las más largas y su acumulación no ha sido inducida, sino directa, es decir, la construcción de $3 \mathrm{~km}$ de costa y, por si no bastara, el terraplenamiento con fines privados, caso de un establecimiento hotelero. El conjunto portuario consta de dos diques: el de Llevant arranca del Postiguet y tiene 2,5 km con dos alineaciones; el de Ponent, 0,7 km perpendicular al Baver. En el interior subsiste la vieja dársena de principios de siglo, cuyo desarrollo fue una réplica del Grau de València. No hace mucho se ganaron terrenos (15 ha) en la parte de poniente, ya muy alterada por las instalaciones portuarias, ferroviarias y fabriles.

Los puertos deportivos, que a menudo se presentan como apoyos a la rururbanización turística, se convierten de hecho en una apropiación de la línea costera para usos privados; así las consecuencias negativas -geomórficas y paisajísticas- son todavía menos justificables que en los grandes puertos. Olvidando la amenaza del superpuerto de Benidorm y otros de la Marina, el Campello contiene tres puertos deportivos (Gozálvez, 1984). Uno está acomodado en el mismo Carrer del Mar y el otro en la Illeta dels Banyets, cuyo paisaje cultural merecería mejor tratamiento.

\section{Paseos marítimos y otros caminos litorales}

Se veía en los primeros una solución colectiva a los problemas de accesibilidad de la orilla marítima y no tanto los inconvenientes que la motorización supone para los teóricos «paseantes», porque paseos peatonales existen bien pocos.

Ejemplos de tramos de una longitud superior a un kilómetro: el Carrer del Mar del Campello, la Platja de Sant Joan y Santa Pola de l'Est. A veces, con la excusa del paseo, se han eliminado cordones de dunas o se han «aprovechado» las restingas.

También se da el caso de carreteras amenazadas por el oleaje. Entre Alacant y el Saladar d'Aigua Amarga la vieja carretera -ahora autovía de $4 \mathrm{~km}$ - tenía tramos comprometidos por los temporales violentos; la ampliación de la banda de rodamiento ha traído consigo una artificialización casi total de la costa, preparada por el ferrocarril. Así han sucumbido yacimientos pleistocenos muy interesantes. Lo mismo cabría decir del antiguo ferrocarril y camino litoral de l'Albufereta: el paisaje litoral -abstrayendo incluso de la edificación- es irreconocible.

\section{Obras de defensa y «regeneración»}

Nueve de cada diez veces, la defensa no va contra la naturaleza, es decir, contra una erosión o un aterramiento espontáneos, sino ingenio contra ingenio. El hombre intenta corregir en un punto el desequilibrio provocado por el prójimo en un litoral cercano. Por la misma causa se habla a menudo de regeneración y pocas veces de generación de playas.

Escolleras y diques constituyeron el clásico recurso defensivo, p.e. en el Baver y Sant Gabriel a lo largo de $1 \mathrm{~km}$ y en el Saladar d'Aigua Amarga, casi $2 \mathrm{~km}$. Pero actualmente se hace más visible la plaga de los espigones y martillos. En el Carrer del Mar del Campello una concesión de cinco espigones de defensa $(1.650 \mathrm{~m})$ se convirtió de hecho en un puerto, como sucedió con los dos diques transversales de Cala Baeza, más al N. Sobre las playas de la Serra Grossa-l'Albufereta, artificiales y separadas por espigones de escollera desiguales y burdos, con un trasfondo de concentración inmobiliaria increíble, todavía se 
querían recrecer con otra generación de defensas y un «paseo marítimo mixto» (1976 ca.). Bien cerca, el Ayuntamiento de Alacant consiguió paralizar otro proyecto semejante más avanzado y especulativo.

\section{Otros impactos negativos}

Todavía podrían enumerarse otras consecuencias desfavorables de la presencia o actividad humana. Ahí están las canteras de piedra de construcción (caliza y tosca) de los alrededores de la ciudad, sobre todo marinos. Recuérdese la recolección tradicional de las falsas algas o posidonias en las playas, e incluso de arena para labores agrícolas. Y la extracción de áridos en los cauces de ríos y ramblas, desatada especialmente desde 1960 ó 1970.

Aún cabe enumerar el desagradable problema de los efluentes urbanos. Alcantarillado y depuración son cuestiones que están lejos de contar con una solución total y definitiva. Los riesgos de contaminación producidos por las congestiones de viviendas litorales están a la vista.

$$
* * *
$$

El hombre resulta ser el principal factor de «descontrol» de las formas litorales (Sanjaume, 1985), sobre todo a causa de las obras concebidas egoísticamente. Término contra término, urbanización contra urbanización, dique contra dique... forman una cadena de actuaciones imparable y esterilizadora. Sería hora de hablar de responsabilidades: el transporte de la deriva litoral es un capital común que no se puede privatizar.

¿Puede esperarse un cambio de óptica? Desde el Plan Indicativo de Usos del Litoral de 1976 a la Política de Costas del MOPU para 1983-90 hay un giro considerable en busca de acciones cuanto menos agresivas mejor. La nueva Ley de Costas, aunque rebajada en su espíritu, permite abrigar esperanzas. La feroz resistencia que ha despertado en el sector más recalcitrante de los especuladores es, a mi parecer, su mejor legitimación.

\section{Bibliografía}

ALBEROLA, A. (1989): «La bonificación de enclaves insalubres en el País Valenciano. El ejemplo de la laguna de la Albufereta (Alicante)». Investigaciones geográficas, 7: 69-81.

GOZÁLVEZ, V. (1984): «La rururbanización del litoral survalenciano». Jornadas de Geografía y Urbanismo. Salamanca, Junta de Castilla y León. Cf. pp. 181-190.

-(1985): «Precisiones sobre los depósitos cuaternarios en la antigua albufera del Saladar de Alicante» Geomorfología litoral y Cuaternario. Homenaje a Juan Cuerda. Universitat de València. Cf. pp. 35-52.

LÓPEZ GÓMEZ, A. y ROSSELLÓ, V. M. (1978): Geografía de la provincia de Alicante. Diputación Provincial de Alicante.

ROSSELLÓ, V. M. (1969): El litoral valencià. I. El medi físic i humà. València, L'Estel. -(1985): «El Pleistocè marí valencià. Història de la seva coneixença», Geomorfología litoral y Cuaternario. Homenaje a Juan Cuerda. Universitat de València. Cf. pp. 135-174. -(1986): «L'artificialització del litoral valencià». Cuad. de Geogr., 38: 1-28.

SANJAUME, E. (1985): Las costas valencianas. Sedimentología y morfología. Universitat de València. 